CARNETS DE Carnets de géographes

GÉOGRAPHES.

2| 2011

Espaces virtuels

\title{
Espaces virtuels
}

\section{Margot Beauchamps et Henri Desbois}

\section{(2) OpenEdition}

Journals

Édition électronique

URL : http://journals.openedition.org/cdg/2617

DOI : $10.4000 /$ cdg. 2617

ISSN : 2107-7266

Éditeur

UMR 245 - CESSMA

Référence électronique

Margot Beauchamps et Henri Desbois, «Espaces virtuels », Carnets de géographes [En ligne], 2 | 2011 , mis en ligne le 02 mars 2011, consulté le 25 septembre 2020. URL : http://journals.openedition.org/ cdg/2617 ; DOI : https://doi.org/10.4000/cdg.2617

\section{(c) (i) (9)}

La revue Carnets de géographes est mise à disposition selon les termes de la Licence Creative Commons Attribution - Pas d'Utilisation Commerciale - Pas de Modification 4.0 International. 


\title{
ESPACES VIRTUELS
}

\author{
MARGOT BEAUCHAMPS \\ Doctorante en géographie à l'université de Paris 1 \\ UMR géographie-cités, Equipe CRIA \\ HENRI DESBOIS \\ Maître de Conférences en géographie \\ Université Paris Ouest Nanterre La Défense
}

Pour ce deuxième numéro des Carnets de géographes, nous abordons un domaine, les espaces virtuels, qui se trouve encore aux marges de la discipline. Les espaces virtuels ne doivent pas seulement s'entendre comme les simulations en 3D interactives telles qu'on les rencontre dans les jeux vidéo et autres réalités virtuelles; ils peuvent aussi s'étendre à la part informationnelle de plus en plus importante de nos environnements depuis nos villes baignées d'informatique jusqu'à certains espaces des Suds où les smartphones permettent une large diffusion de l'Internet.

Les loisirs, le travail, les transports dépendent de plus en plus de l'informatique à tel point que certains géographes vont jusqu'à parler d'un « espace de code » qui nous contrôle, qui nous gouverne et dans lequel nous sommes plongés à chaque moment de la vie (Graham, 2005).

C'est à ce titre que notre espace quotidien est de plus en plus, au moins en partie, un espace virtuel. Depuis qu'en 1984, le romancier William Gibson a popularisé le mot dans son roman Neuromancien, le terme «cyberespace », dont le succès est tel qu'il est passé dans le vocabulaire courant pour désigner l'Internet, suggère une spatialité effective du monde virtuel, et non simplement métaphorique comme on le suppose souvent. Nous proposons dans ce deuxième numéro des Carnets de géographes de prendre au sérieux la spatialité du virtuel et d'inviter les géographes à investir cette nouvelle espèce d'espace. C'est ce à quoi s'est attelé Boris Beaude qui retrace, dans un entretien publié dans ces Carnets de débats, le cheminement de sa réflexion sur la spatialité de l'Internet. 
La géographie comme les autres sciences sociales doivent prendre en compte cette part invisible mais désormais essentielle de nos territoires. Parce qu'elles sont invisibles et qu'elles sont jeunes, nous avons encore du mal à appréhender ces technologies dans leur globalité et à mesurer leurs influences.

La science fiction, dans ce domaine en avance sur les sciences sociales, a souvent mis en scène le virtuel. Par des expériences de pensée, par des allégories, les auteurs de science fiction ont exploré le potentiel des techniques numériques parfois avant même leur apparition. Ainsi, un détour par la science fiction peut être fécond pour penser les transformations opérées par les techniques numériques. En explorant ces objets, la science fiction, qu'elle soit prophétique ou trompeuse contribue à fabriquer le prisme à travers lequel nous percevons, concevons et imaginons la part virtuelle de notre environnement.

Les articles présentés dans ce numéro montrent la diversité des thèmes et des approches des espaces virtuels. Ce numéro a souhaité s'appuyer sur les contributions de la journée d'étude "Réalité des espaces virtuels » organisée conjointement par l'EHESS et l'équipe Réseaux, Savoirs et Territoires le 10 juin 2010, tout en élargissant la réflexion à de nouvelles perspectives.

Les articles directement issus de la journée d'étude sont regroupés dans les Carnets de recherche et présentent différentes approches théoriques et des espaces virtuels comme objet de recherche en les rattachant à la science fiction et aux jeux vidéo. Alain Musset montre comment la science fiction produit une lecture du monde dont la géographie peut tirer profit.

Le cyberespace de la science fiction a fourni un imaginaire puissant au monde des ingénieurs, puis au grand public, imaginaire qui façonne notre vision de notre monde et de la ville. C'est ce que montre Henri Desbois dans son Carnet de recherches.

À travers une typologie des espaces virtuels, l'article de Guy Thuillier aboutit au constat d'une contamination du réel par le virtuel et contribue ainsi à dépasser l'opposition entre mondes réels et mondes virtuels en assumant la réalité des espaces virtuels qui occupent une place prépondérante dans notre imaginaire et notre quotidien.

Avec une approche sociologique, Laurent Tremel montre que les interactions structurées par les jeux vidéo reproduisent les enjeux de pouvoir et des stratifications sociales du monde matériel. 
Ces approches sont complétées dans les Carnets de terrain par deux articles théoriques qui posent la question de la légitimité de la recherche sur ces objets marginaux et deux exemples de la manière dont les terrains virtuels peuvent s'intégrer dans la pratique de l'enseignant et du chercheur.

Les deux articles théoriques présentent les difficultés spécifiques que posent les espaces virtuels comme objet d'étude et s'attachent à anticiper les soupçons que suscitent le fait de se pencher sur un objet jugé a priori peu sérieux (les jeux vidéo pour Samuel Ruffat et Hovig Ter Minassian) ou éloigné du terrain comme l'entend la tradition géographique dominante (Internet, pour Boris Baude).

En s'appuyant sur un récit semi-fictif, Anthony Merle s'interroge sur les apports d'une utilisation pédagogique du terrain virtuel à travers Google Earth. Enfin, à partir du cas spécifique de l'étude des impacts des acteurs miniers dans une région aurifère du Burkina Faso, Quentin Megret montre comment le travail sur le terrain «matériel » doit être complété par une analyse des constructions virtuelles des acteurs, institutions et entreprises, dont une partie des stratégies et comportements est invisible sur le terrain matériel.

L'étude des espaces virtuels est encore un peu jeune pour qu'il en existe une véritable tradition. La variété des approches dont témoigne ce numéro donne un aperçu de la richesse de ce nouveau terrain sur lequel on souhaite que les géographes osent davantage s'aventurer.

\section{Référence bibliographique}

GRAHAM S., 2005, «Software-sorted Geographies», Progress in Human Geography 29, (5) pp. 562-580 